\title{
Serial Frozen Fecal Microbiota Transplantation in the Treatment of Chronic Intestinal Pseudo-obstruction: A Preliminary Study
}

\author{
Lili Gu, Chao Ding, Hongliang Tian, Bo Yang, Xuelei Zhang, Yue Hua, Yifan Zhu, Jianfeng Gong, Weiming Zhu, Jieshou Li, and \\ Ning $\mathrm{Li}^{*}$ \\ Department of General Surgery, Jinling Hospital, Nanjing University School of Medicine, Nanjing; China
}

\begin{abstract}
Background/Aims
Chronic intestinal pseudo-obstruction (CIPO) is a serious, life-threatening motility disorder that is often related to bacterial overgrowth. Fecal microbiota transplantation (FMT) results in restoration of the normal intestinal microbial community structure. We investigated the efficacy of FMT in the treatment of CIPO patients.

\section{Methods}

Nine patients (age 18-53 years) with CIPO were enrolled in this prospective, open-label study. Patients received FMT for 6 consecutive days through nasojejunal (NJ) tubes and were followed up for 8 weeks after treatment. We evaluated the rate of clinical improvement and remission, feeding tolerance of enteral nutrition, and CT imaging scores of intestinal obstructions. Lactulose hydrogen breath tests were performed before FMT and 8 weeks after FMT to evaluate for the presence small intestinal bacterial overgrowth (SIBO).
\end{abstract}

\section{Results}

FMT significantly alleviated bloating symptoms, and symptoms of pain were relieved 2 weeks after FMT. Enteral nutrition administered through a NJ tube after FMT was well-tolerated by $66.7 \%$ (6/9) of patients. CT scores of intestinal obstructions were significantly reduced after FMT $(P=0.014)$. SIBO was eliminated in $71.0 \%(5 / 7)$ of patients.

\section{Conclusions}

This pilot study demonstrated the safety of using FMT. FMT may relieve symptoms in selected patients with CIPO. FMT may also improve patient tolerance of enteral nutrition delivered via a NJ tube.

(J Neurogastroenterol Motil 2017;23:289-297)

Key Words

Intestinal pseudo-obstruction; Fecal microbiota transplantation; Tolerance of enteral nutrition

Received: May 15, 2016 Revised: October 9, 2016 Accepted: October 13, 2016

(.) This is an Open Access article distributed under the terms of the Creative Commons Attribution Non-Commercial License (http://creativecommons. org/licenses/by-nc/4.0) which permits unrestricted non-commercial use, distribution, and reproduction in any medium, provided the original work is properly cited.

*Correspondence: Ning Li, PhD

Department of General Surgery, Jinling Hospital, Nanjing University School of Medicine, No. 305 East Zhongshan Road, Nanjing 210002, China

Tel: +86-025-80860089, Fax: +86-025-80860089, E-mail: liningrigsnju@163.com 


\section{Introduction}

Intestinal pseudo-obstruction can be categorized as either acute or chronic. Chronic intestinal pseudo-obstruction (CIPO) is a rare clinical syndrome first reported by Dudley et $\mathrm{al}^{1}$ in 1958. CIPO is a functional motility disorder characterized by episodic obstructive symptoms in the absence of a mechanical cause. In most cases, operative treatment cannot circumvent the course of CIPO and can actually worsen the motility disorder. ${ }^{2}$ Inadequate oral intake, recurrent episodes of obstruction, and malabsorption are the main clinical features of CIPO, with $20 \%$ to $60 \%$ of patients needing long-term home parenteral nutrition. ${ }^{3}$ Thus, the main goals in CIPO management are improvement of intestinal propulsion and maintenance of adequate nutritional status. ${ }^{4}$ Recently, the ability to maintain oral feeding was found to be an independent predictor of survival in patients with $\mathrm{CIPO}^{2}$

The etiology and cause of CIPO are not well clarified. When impairment of gut motility and transit are severe, small intestinal bacterial overgrowth (SIBO) may occur, further contributing to malnutrition and vitamin deficiency. ${ }^{5}$ SIBO may also in turn exacerbate intestinal dysmotility. ${ }^{6,7}$ Currently, non-absorbable antibiotics (eg, rifaximin) are used to alleviate SIBO-associated symptoms. This beneficial effect of antibiotics may in part be due altered location or quality of the patient's gastrointestinal microbiota. ${ }^{10}$

Fecal microbiota transplantation (FMT) is a technique in which intestinal microbiota are transferred from a healthy donor to a patient with altered gut microbiota because of a disease or condition. The goal of FMT is to treat the patient by replicating the phylogenetic microbiota diversity found in the healthy donor. Most clinical experience with FMT to date has been in relation to the treatment of Clostridium difficile infections. ${ }^{11,12}$ However, FMT may also be effective in the treatment of other conditions associated with dysbiosis, such as inflammatory bowel disease, irritable bowel syndrome (IBS), and metabolic syndrome. Although the exact mechanism of the effect of FMT remains unclear, some research suggests that alleviation of symptoms after FMT is associated with changes in microbial community structure, such as restoration of microbial diversity. ${ }^{13}$

Because gut microbiota dysbiosis contributes to the pathology of CIPO, we hypothesized that manipulating the gut microbiome through FMT could reverse intestinal dysbiosis and consequently resolve overt CIPO. The present pilot study aim to evaluate the efficacy and safety of FMT delivered via a nasojejunal (NJ) tube for the treatment of CIPO. We also present a simple and easily repro- ducible protocol to identify and screen stool donors and to perform the FMT.

\section{Materials and Methods}

\section{Patients}

The study sample comprised of 9 patients ( 5 women, median age 32 years) with a long-standing diagnosis of CIPO (median duration of illness 35 months). Eligible CIPO patients were identified via specialist gastrointestinal clinics. To be eligible, patients had to meet the diagnostic criteria for CIPO proposed by the Japanese Ministry of Health, Labour, and Welfare, ${ }^{14}$ including documented pathological bowel dilatation in the absence of mechanical obstruction on imaging. The patients underwent FMT between December 2014 and September 2015.

\section{Lactulose Hydrogen Breath Test}

A lactulose hydrogen breath test was administered to patients before and after FMT to assay for the presence of SIBO. Patients were instructed to fast for at least 12 hours before the test. Patients were asked to brush their teeth and rinse out their mouths with an antiseptic mouth wash at least 2 hours before the test to avoid false positive high basal levels of hydrogen from fermentation of substrate by oral bacteria. Patients were administered d $75 \mathrm{~g}$ of lactose in 150 $\mathrm{mL}$ of water, and breath samples were collected every 30 minutes for 3 hours. Patients were asked to refrain from any exercise, sleep, or cigarette smoking during the test to avoid possible effects on breath hydrogen levels. Breath concentrations of hydrogen and methane were analyzed immediately by gas chromatography using an SC Microlyzer (QuinTron Instrument Co, Milwaukee, WI, USA). The results were reported in parts per million (ppm). SIBO was diagnosed if breath concentrations of hydrogen or methane were $\geq 12$ ppm over baseline values for at least 3 measurements. ${ }^{15}$

\section{Fecal Donors}

Volunteer fecal donors were prospectively screened and rescreened every 6 months according to recommendations by Bakken et al. ${ }^{16}$ Donor feces were tested for enteric pathogens, including Salmonella, Shigella, Campylobacter, Escherichia coli O157 H7, Yersinia, C. difficile toxin, helminths, ova, cysts, parasites, and $\mathrm{He}$ licobacter pylori. Fecal testing was performed using standard methods. Most stools were obtained from 4 donors (age, 20-24 years) who met the selection criteria. Donors who met the selection criteria donated stool samples at least 48 hours before the FMT to permit 
preparation of samples.

\section{Preparation of Fecal Suspensions}

Approximately $100 \mathrm{~g}$ of fresh stool was mixed with $500 \mathrm{~mL}$ of $0.9 \%$ sterile saline for several seconds to achieve a smooth consistency. To strain out large particles, the sample was filtered through a gauze placed on top of an empty container. A portion of the filtered suspension $(100 \mathrm{~mL})$ was transferred into an enema bag. The rest of the suspension $(150 \mathrm{~mL})$ was diluted in $0.9 \%$ sterile saline to a volume of 400-425 mL and filtered through a series of gauze screens $(2.0 \mathrm{~mm}$ to $0.7 \mathrm{~mm}$ to $0.2 \mathrm{~mm}$ ) to remove small particles. The resulting concentrated fecal suspension was amended with glycerinum to a final concentration of $10 \%$ and stored at $-20^{\circ} \mathrm{C}$ for a maximum of 30 days. The stool suspension was thawed at $25^{\circ} \mathrm{C}$, poured into a sterile bottle, and used for FMT within 2 hours.

\section{Fecal Microbiota Transplantation Procedure}

First, a NJ tube was placed in the patient's proximal jejunum endoscopically or via X-ray fluoroscopy, and the position of the tube was verified by abdominal radiography. To confirm the absence of any mechanical obstruction, $100 \mathrm{~mL}$ of undiluted contrast agent (Gastrografin, Bayer Schering Pharma, Germany) was administered through the NJ tube by a doctor who was not associated with the study. After flatus or defecation, the patient was administered $500 \mathrm{mg}$ vancomycin through the NJ tube once per day for 3 consecutive days. Following antibiotic treatment, the prepared stool suspension was delivered through the NJ tube for 6 consecutive days. Patients were fasted and were given either parenteral nutrition or intravenous fluids during the FMT treatment.

\section{Efficacy and Outcomes}

Primary endpoints were as follows: (1) the tolerance of enteral nutrition (EN) through the NJ tube. Feeding tolerance (FT) was defined as the ability to deliver $80 \%$ of the amount of nutrient as a proportion of energy requirements, with values of $80 \%$ chosen or a certain volume $(750 \mathrm{~mL})$ per 24 hours $^{17}$ and (2) the ability to eat food and finish a meal (yes/no). The start of oral intake (both fluids and solid food) was also noted.

Secondary endpoints were symptomatic relief and scoring evaluation of the abdominal CT. Patients were required to keep a daily diary of the occurrence and severity of pain, nausea, vomiting, and bloating using the following scores: $0=$ absent, $1=$ mild, 2 $=$ medium, $3=$ severe, or $4=$ could not be worse. These data were subsequently recorded by a nurse who was not associated with the FMT procedure. A CT scoring system ${ }^{18}$ was used to evaluate the severity of obstruction before and after FMT (Table 1). This system scores the need for surgery based on CT findings of free air, transition points, complete obstruction, closed loops, free fluid, and partial obstruction. Abdominal CT was conducted before FMT and at 8 weeks after FMT, with scores calculated as "initial CT findings" and "repeat CT findings," respectively. A score of 8 or higher predicted the need for surgery $75 \%$ of the time, and a value of 8 on the scoring system affects the sensitivity versus specificity. ${ }^{18}$

After completing 9 days of treatment, patients were evaluated for 8 additional weeks. Study visits were conducted on the third day of FMT and on days 7, 14, 21, 28, and 56. Efficacy assessments were obtained daily via an interactive voice-response system over the course of the entire study.

\section{Adverse Events Reporting}

Patients recorded any adverse events in their daily diary and reported them to the investigator. The investigator determined the likelihood of the reported adverse event being related to the study treatments.

\section{Statistical Methods}

The primary endpoint was the duration (in days) of symptoms until patients could tolerate EN through the NJ tube. All statistical analyses were performed using SPSS version 22.0 (SPSS, Inc, Chicago IL, USA). Non-normally distributed data are presented as medians and quartiles (25th and 75th percentiles), and categorical data are presented as n (\%). Outcomes before and after treatment were compared using the Wilcoxon rank sum test. $P<0.05$ was considered to be statistically significant.

Table 1. Point Values for Computed Tomography Scoring System

\begin{tabular}{ll}
\hline Initial CT findings & 5 \\
Free air & 3 \\
Transition point & 3 \\
Complete obstruction & 3 \\
Closed loop & 3 \\
Free fluid & 2 \\
Partial obstruction & \\
Repeat CT findings & -5 \\
Resolution & -2 \\
Improved obstruction & +2 \\
Persistent SBO & +3 \\
Worsening obstruction & +5 \\
Free air &
\end{tabular}

SBO, small-bowel obstruction

A score of 8 or higher predicted the need for surgery $75 \%$ of the time, a value of 8 on the scoring system affects the sensitivity versus specificity. 


\section{Ethics}

Patients were fully informed of the benefits and potential risks of standardized FMT and laboratory screening. Written, informed consent was obtained from all patients. The research protocol was approved by the Jinling Hospital's medical ethics committee and was registered at ClinicalTrials.gov (NCT 02731183). All patients provided written informed consent.

\section{Results}

\section{Patient Characteristics}

Characteristics of the 9 patients with CIPO who received FMT are shown in Table 2.

\section{Effect of Fecal Microbiota Transplantation on Enteral Nutrition Feeding Tolerance and Ability to Eat Food}

At 8 weeks after FMT, 6 of $9(66.7 \%)$ patients could tolerate EN through an NJ tube; most of the estimated caloric needs of these patients were satisfied, and they did not require parenteral nutrition. Although 3 patients $(33.3 \%)$ patients still exhibited feeding intolerance and required home parenteral nutrition, 4 (44.4\%) were able to eat normally 8 weeks after FMT. Two patients (patients 5 and 9) received home enteral nutrition support.

\section{Fecal Microbiota Transplantation Effects on Symptom Evaluations, Computed Tomography Scoring, and Lactulose Hydrogen Breath Test Results}

FMT affected the CIPO symptom severity. A significant timeby-treatment interaction was observed for the bloating and pain

Table 2. Demographic Data of Fecal Microbiota Transplant Recipients

\begin{tabular}{|c|c|c|c|c|c|c|c|c|}
\hline Patient & Sex & Age & $\begin{array}{l}\text { Weight } \\
\text { (kg) }\end{array}$ & $\begin{array}{c}\text { BMI } \\
\left(\mathrm{kg} / \mathrm{m}^{2}\right)\end{array}$ & $\begin{array}{l}\text { Serum pre-albumin } \\
(\mathrm{mg} / \mathrm{L})\end{array}$ & $\begin{array}{l}\text { Creatinine } \\
(\mu \mathrm{mol} / \mathrm{L})\end{array}$ & $\begin{array}{l}\text { Time since onset of } \\
\text { symptoms (mo) }\end{array}$ & $\begin{array}{l}\text { Other treatments } \\
\text { before FMT }\end{array}$ \\
\hline 1 & $\mathrm{~F}$ & 17 & 35.0 & 13.6 & 212 & 36 & 124 & Prucalopride \\
\hline 2 & $\mathrm{~F}$ & 33 & 48.5 & 19.4 & 160 & 50 & 112 & Partial intestine resection \\
\hline 3 & M & 16 & 38.0 & 14.0 & 74 & 60 & 24 & $\begin{array}{l}\text { Operation of intestine } \\
\text { adhesion relaxation }\end{array}$ \\
\hline 4 & M & 28 & 43.0 & 13.0 & 68 & 208 & 35 & Ileostomy \\
\hline 5 & $\mathrm{~F}$ & 18 & 28.0 & 11.4 & 190 & 22 & 22 & Mosapride \\
\hline 6 & $\mathrm{~F}$ & 29 & 45.0 & 17.3 & 179 & 59 & 13 & Ileostomy \\
\hline 7 & M & 32 & 56.0 & 17.6 & 225 & 69 & 12 & Ileostomy \\
\hline 8 & $\mathrm{~F}$ & 50 & 56.0 & 15.5 & 155 & 51 & 24 & Ileostomy \\
\hline 9 & M & 68 & 59.0 & 19.3 & 146 & 54 & 8 & None \\
\hline
\end{tabular}

BMI, body mass index; FMT, fecal microbiota transplant; F, female; M, male.

Table 3. Changes of Symptoms Scores of 9 Patients Completing the Study at Different Time Points

\begin{tabular}{|c|c|c|c|c|c|c|c|c|}
\hline & \multicolumn{2}{|l|}{ Bloating } & \multicolumn{2}{|l|}{ Nausea } & \multicolumn{2}{|l|}{ Pain } & \multicolumn{2}{|l|}{ Vomiting } \\
\hline & $\begin{array}{c}\text { Medians (lower and } \\
\text { upper quartiles) }\end{array}$ & $P$-value & $\begin{array}{c}\text { Medians (lower and } \\
\text { upper quartiles) }\end{array}$ & $P$-value & $\begin{array}{c}\text { Medians (lower and } \\
\text { upper quartiles) }\end{array}$ & $P$-value & $\begin{array}{l}\text { Medians (lower and } \\
\text { upper quartiles) }\end{array}$ & $P$-value \\
\hline Before FMT & $3(2.5,4.0)$ & & $2(0.5,2.0)$ & & $2(1.0,2.5)$ & & $2(0.5,3.0)$ & \\
\hline Day 6 & $2(2.0,3.0)$ & $0.025^{\mathrm{a}}$ & $2(1.5,2.5)$ & 0.180 & $2(1.0,2.5)$ & $>0.99$ & $2(1.0,3.0)$ & 0.705 \\
\hline $1 \mathrm{wk}$ & $2(2.0,3.0)$ & $0.011^{\mathrm{a}}$ & $2(1.0,3.0)$ & 0.083 & $1(1.0,2.5)$ & 0.157 & $2(1.0,2.5)$ & 0.655 \\
\hline $2 \mathrm{wk}$ & $2(1.0,2.0)$ & $0.009^{\mathrm{a}}$ & $2(1.0,2.0)$ & $>0.99$ & $1(0.5,2.0)$ & $0.014^{\mathrm{a}}$ & $1(1.0,2.0)$ & 0.257 \\
\hline $3 \mathrm{wk}$ & $1(1.0,2.0)$ & $0.010^{\mathrm{a}}$ & $2(0.5,2.0)$ & 0.564 & $1(0.0,1.5)$ & $0.023^{\mathrm{a}}$ & $1(0.5,2.0)$ & 0.157 \\
\hline $4 \mathrm{wk}$ & $1(1.0,3.0)$ & $0.011^{\mathrm{a}}$ & $1(0.0,2.0)$ & 0.250 & $1(0.0,1.5)$ & $0.023^{\mathrm{a}}$ & $1(0.0,2.0)$ & $0.020^{\mathrm{a}}$ \\
\hline $8 \mathrm{wk}$ & $1(0.0,3.0)$ & $0.010^{\mathrm{a}}$ & $1(0.0,2.0)$ & 0.630 & $1(0.0,1.5)$ & $0.014^{\mathrm{a}}$ & $1(0.0,2.0)$ & 0.084 \\
\hline
\end{tabular}

${ }^{a} P<0.05$, statistically significant reduction of symptoms vs before treatment.

FMT, fecal microbiota transplant.

Each group of statistics are indicated with medians and quartiles ( 25 th percentile, 75 th percentile). Scores represented as $0=$ absent, $1=$ mild, $2=$ medium, $3=$ severe, or $4=$ could not be worse. 
symptom scores. As shown in Table 3, bloating scores demonstrated a significant reduction among data obtained at baseline, 6 days median 2 (lower quartile: 2, upper quartile: 3), 1 week $2(2,3), 2$ weeks $2(1,2), 3$ weeks $1(1,2), 4$ weeks $1(1,3)$, and 8 weeks $1(0,3)$ $(P<0.05)$. The patient's pain scores were reduced from baseline $2(1,2.5)$ to 2 weeks $1(0.5,2), 3$ weeks $1(0,1.5), 4$ weeks $1(0$, $1.5)$, and 8 weeks $1(0,1.5)(P<0.05)$. The vomiting scores were reduced only in 4 weeks $1(0,2)$ from baseline $2(0.5,3)(P<0.05)$. No significant difference was found between pre- and post-FMT nausea scores.
CT scores of the 9 patients are shown in Table 4. All scores were under 8, and the scores of repeat CT findings (median: 3, range: 0-5) compared with initial CT findings (median: 5, range: $2-6$,$) were significantly reduced (P=0.014)$. These data suggest that a significant number of patients were in remission following FMT.

Pre- and post-FMT plain radiographs and abdominal CT scans of patient 3 are shown in Figures 1 and 2, respectively.

Lactulose hydrogen breath testing showed that 7 of 9 patients $(77.8 \%)$ were positive for SIBO at baseline. However, 8 weeks

Table 4. Clinical Course of Fecal Microbiota Transplant Recipients

\begin{tabular}{|c|c|c|c|c|c|c|c|}
\hline Patient & $\begin{array}{c}\text { Primary clinical } \\
\text { outcome }^{\mathrm{a}}\end{array}$ & $\begin{array}{c}\text { Time since FMT } \\
(w \mathrm{k})^{\mathrm{b}}\end{array}$ & $\begin{array}{l}\text { Ability of } \\
\text { oral intake }\end{array}$ & $\begin{array}{c}\text { CT score } \\
\text { (initial CT findings) }\end{array}$ & $\begin{array}{c}\text { CT score } \\
\text { (repeat CT findings) }\end{array}$ & $\begin{array}{c}\text { SIBO } \\
\text { (before FMT) }\end{array}$ & $\begin{array}{c}\text { SIBO } \\
(\text { after FMT) }\end{array}$ \\
\hline 1 & - & & - & 6 & 4 & + & + \\
\hline 2 & + & 6.0 & + & 5 & 0 & + & - \\
\hline 3 & + & 5.5 & + & 6 & 1 & + & - \\
\hline 4 & - & & - & 5 & 5 & - & - \\
\hline 5 & + & 4.0 & - & 5 & 3 & + & - \\
\hline 6 & + & 5.0 & + & 2 & 0 & + & + \\
\hline 7 & - & & - & 6 & 4 & - & - \\
\hline 8 & + & 7.0 & + & 2 & 2 & + & - \\
\hline 9 & + & 4.0 & - & 6 & 4 & + & - \\
\hline
\end{tabular}

${ }^{a}$ Primary endpoint: the tolerance of enteral nutrition through nasojejunal tube 8 weeks after fecal microbiota transplant (FMT). We defined feeding tolerance as ability to deliver the amount of nutrient as a proportion of "energy requirements," with values of $80 \%$ chosen, or a certain amount (750 mL) per 24 hours.

${ }^{\mathrm{b}}$ Time from FMT to tolerate enteral nutrition.

${ }^{\mathrm{c}} \mathrm{P}<0.05$ (repeat CT findings vs initial CT findings).

SIBO, small bowel bacterial overgrowth; -, indicates not applicable; + , applicable.

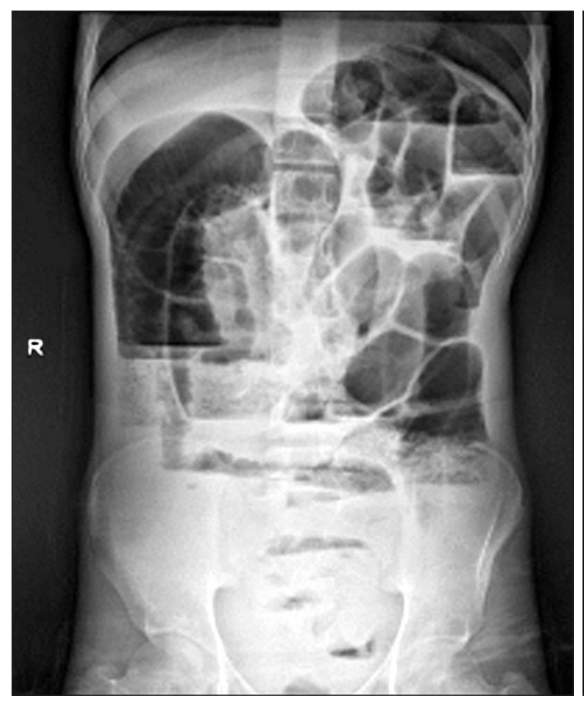

Before treatment

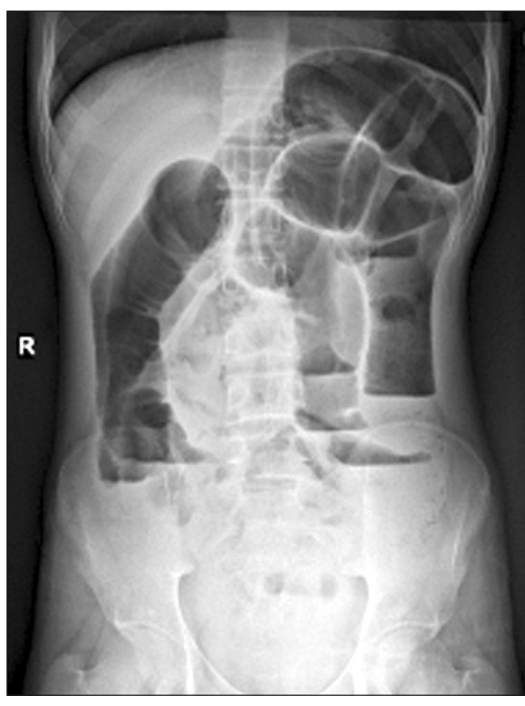

During treatment

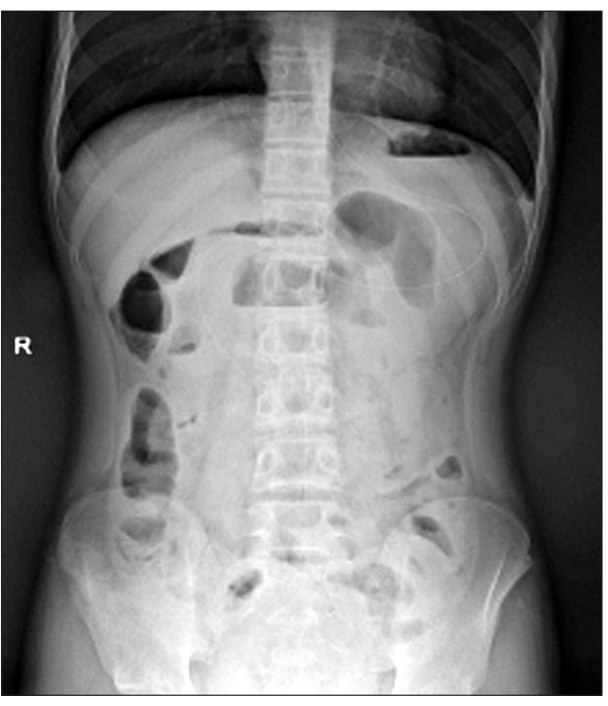

After treatment

Figure 1. X-ray plain films of Patient 3 at different periods. Abdominal plain radiograph performed in upright position shows multiple distended bowel loops before treatment. No mechanical cause of obstruction is seen. This situation improved significantly after fecal microbiota transplant treatment for 8 weeks. R, right. 

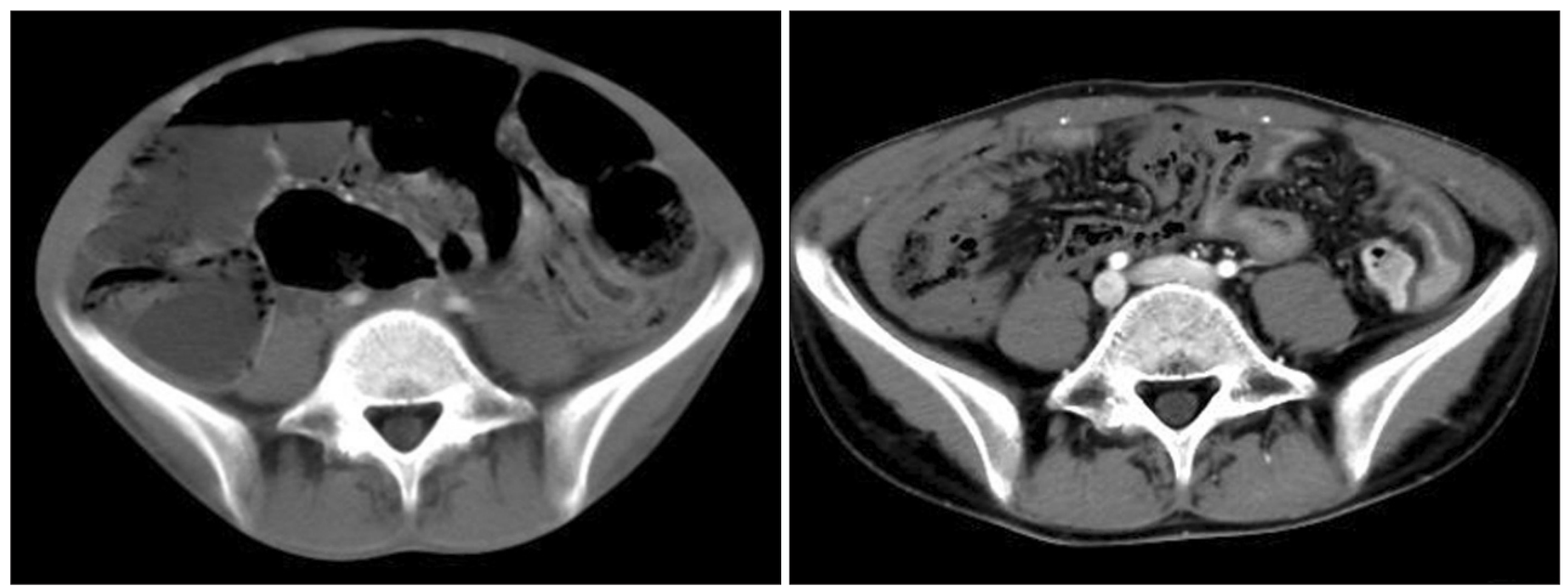

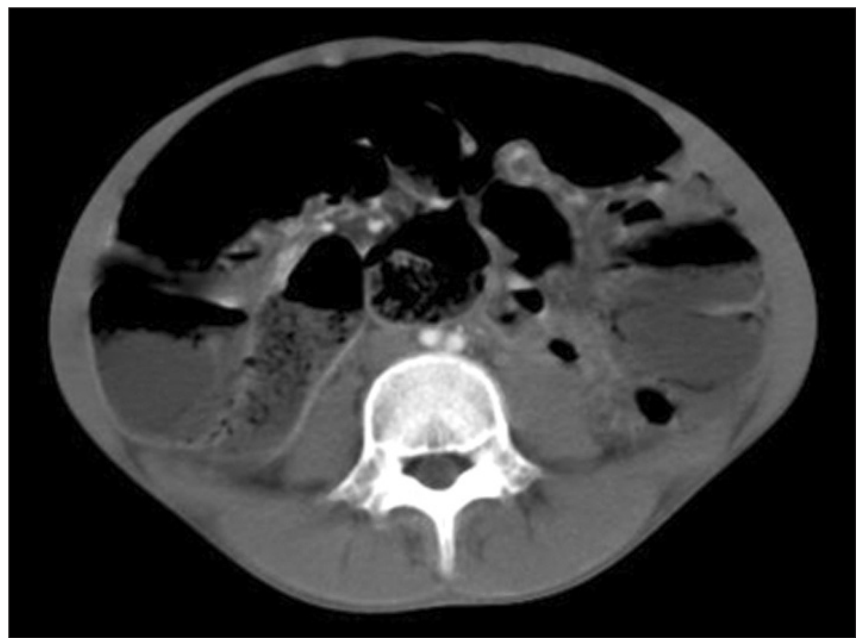

Before treatment

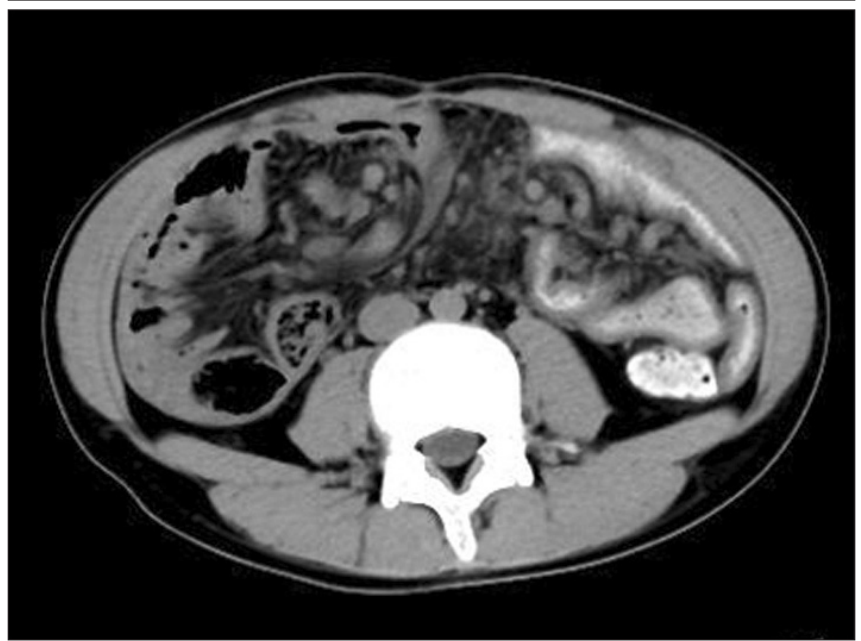

8 weeks after FMT

Figure 2. CT scan of patient 3 at different periods. Axial CT images obtained at different levels of slice show marked distension and gas-fluid levels. No mechanical cause of obstruction is seen. These symptoms disappeared after serial fecal microbiota transplant (FMT) treatment.

after FMT, only 2 patients (patients 1 and 6) tested positive for SIBO (Table 4). These findings indicate that SIBO was resolved in $71.0 \%(5 / 7)$ of patients after FMT.

\section{Intolerance and Adverse Events}

No severe adverse events were observed during endoscopic infusion, immediately after FMT, or during the 8-week follow-up period.

\section{Discussion}

CIPO is one of the most severe and well-described gastrointestinal neuromuscular diseases. It is not a single disorder but is rather a heterogeneous group of disorders characterized by disordered peristalsis. In CIPO, the normal antegrade propulsive activity of the intestinal tract is defective, thereby resulting in the development of symptoms, such as abdominal pain, nausea, vomiting, and bloating. Because affected individuals are often unable to maintain adequate nutrition and normal body weight, CIPO is a severe cause of chronic intestinal failure. CIPO is a rare and often misdiagnosed disease. CIPO patients often undergo useless and potentially dangerous abdominal surgeries. Traditional surgical procedures are generally unable to correct the digestive dysmotility characteristic of this disease. ${ }^{19}$

A large percentage of patients with CIPO require different forms of nutritional support. ${ }^{3}$ The long-term outcome is often poor for many CIPO patients because they cannot maintain their nutritional status. Many patients cannot tolerate enteral nutrition, 
whether taken orally or delivered through nasogastric or NJ tubes. About $20 \%$ to $60 \%$ of patients require long-term home parenteral nutrition, and complications related to parental nutrition administration occur in $45-80 \%$ cases. The mortality rate for adults with CIPO is about $10 \%{ }^{20}$ Hence, the goals of treatment include control of symptoms, enhancement in nutritional status, improvement of nutritional status, and maintenance of quality of life. ${ }^{21,22}$

The etiology of primary CIPO is varied. Abnormalities of smooth muscle cells, interstitial cells of Cajal, intrinsic (enteric) and extrinsic neural supplies, and the central nervous system may all play a role in the pathophysiology of CIPO whether separately or in various combinations. ${ }^{23}$ Symptoms can vary depending on which intestinal segment is primarily affected. Abnormal motility is associated with SIBO, which causes mucosal inflammation, thereby directly affecting the sensorimotor function of the intestine and creating a vicious cycle. ${ }^{24}$

SIBO with coliform bacteria is common and well described in patients with CIPO. ${ }^{6,25}$ SIBO may also cause symptoms, such as chronic diarrhea, bloating, abdominal distention, flatulence, and changes in bowel habits. ${ }^{26}$ These symptoms may occur because of increased gas production, toxic byproducts, or increased osmotic load due to bacterial metabolism of substrates in the small intestine. In a study of intestinal motility in patients with $\mathrm{SIBO}^{27} 51 \%$ had altered motility, resulting in symptoms similar to those associated with IBS; eradication of SIBO resulted in normalization of motility. Treatment with poorly absorbed antibiotics, such as rifaximin, may improve motility. ${ }^{28}$ Notably, antibiotic treatment for SIBO may stop diarrhea but can induce a reappearance of constipation, leading to worsening of CIPO symptoms. ${ }^{5}$ A recent study showed that antibiotic treatment may decrease the fecal output, delay whole gut and colonic transit, and reduce spontaneous contractions and the response to acetylcholine in the ileum and colon. ${ }^{29}$ Accordingly, the treatment strategies for SIBO include not only elimination of pathogenic bacteria but also restoration of normal intestinal bacterial microflora, improvement of gastrointestinal motor function, control of visceral sensitivity, and proper nutritional management. ${ }^{30}$

FMT involves administration of fecal material containing distal gut microbiota from a healthy person (donor) to a patient with a disease or condition related to dysbiosis, or to a patient with altered gut microbiota. FMT aims to treat the disease by restoring phylogenetic diversity and microbiota that are typically found in a healthy person. ${ }^{13}$ The most common clinical application of FMT is treating C. difficile infections which are otherwise unresponsive to standard antibiotic therapies. ${ }^{12}$ FMT is becoming more widely practiced and has gained interest of patients, researchers, and industry in recent years. Because alterations in the intestinal microbiota contribute to CIPO, we hypothesized that manipulating the gut microbiome through FMT could reverse intestinal dysbiosis and result in resolution of overt CIPO. Hence, we conducted this pilot study to explore the effect of FMT in CIPO treatment.

There are numerous routes by which FMT can be administered, including infusion via nasogastric or nasoenteric tubes, esophagogastroduodenoscopy (EGD), colonoscopy, and enema. There is no clear consensus on the best method of instillation. FMT is reported to be effective by all of these routes, and the preferred method may vary with the clinical situation. ${ }^{13}$ Because CIPO predominantly affects the small intestine, in our study, an NJ tube was placed using X-ray fluoroscopy or endoscopy. Following tube placement, alimentary tract contrast examination was performed to verify the absence of mechanical obstruction. The non-absorbed antibiotic vancomycin was delivered through the $\mathrm{NJ}$ tube to reduce local gut mucosal engagement of bacteria, and then, previously frozen fecal material was administered to patients through the $\mathrm{NJ}$ tube for 6 consecutive days.

After 8 consecutive weeks of observation following FMT, patients' symptom scores were significantly reduced for pain and bloating but not for vomiting and nausea. Chronic abdominal pain and bloating are 2 predominant symptoms in patients with CIPO. Chronic abdominal pain is defined as an unpleasant sensation that is diffuse and difficult to localize compared with somatic pain. ${ }^{31}$ Gut microbiota dysbiosis, luminal dilatation, increased intestinal permeability, and mucosal inflammation may contribute to the development of visceral hyperalgesia with sensitization of peripheral and central pain pathways. ${ }^{3,32}$ Visceral hypersensitivity characterizing constipation-predominant IBS patients can be transferred to rats by fecal microbiota. ${ }^{33}$ In IBS patients, alteration of intestinal microbiota has been linked to immune dysfunction and altered neurological functions, such as reduced pain threshold and increased anxiety, suggesting that restoration of normal intestinal homeostasis via FMT may result in symptomatic improvement. ${ }^{34}$ Our results confirmed that FMT delivered through an NJ tube may relieve abdominal pain and bloating symptoms in CIPO patients.

The microbiota plays a key role in the development and promotion of normal gut neuromotor function. The etiology of malnutrition in CIPO is multifactorial and includes the following: inadequate intake of nutrients, malabsorption from bacterial overgrowth, and increased metabolic demand secondary to inefficient production of ATP within the mitochondria. ${ }^{35}$ Above all, the intolerance of $\mathrm{EN}$ in CIPO may be due to dysmotility of the digestive tract. In our study, we observed that after serial FMT for 6 consecutive 
days, 6 out of 9 patients could tolerate EN through an NJ tube, with the amount of nutrition meeting $80 \%$ of total energy requirements. Furthermore, 4 out of 9 patients could tolerate an oral liquid diet. These results suggest that serial FMT may be a candidate therapy for CIPO.

This study had several limitations. First, the number of cases in the study was low, and a comparison group was not included. The incidence rates of CIPO are low, and 6 of these patients were surgically treated, 4 of whom underwent ileostomy. Thus, the individual difference is obvious. Second, follow up for this study was only out to 8 weeks. A longer follow-up time is necessary to assess the longterm efficacy of FMT. Finally, the composition of the intestinal microbiota was not investigated before and after FMT. The changes in the abundance and diversity of intestinal microorganisms following FMT need to be examined. Randomized trials will be necessary to answer the many challenging questions regarding clinical applications of this treatment.

In summary, the results of this pilot study suggest that FMT may be beneficial for the treatment of CIPO. Carefully designed randomized trials will be needed to confirm the findings.

\section{References}

1. Dudley HA, Sinclair IS, McLaren IF, McNair TJ, Newsam JE. Intestinal pseudo-obstruction. J R Coll Surg Edinb 1958;3:206-217.

2. Amiot A, Joly F, Cazals-Hatem D, et al. Prognostic yield of esophageal manometry in chronic intestinal pseudo-obstruction: a retrospective cohort of 116 adult patients. Neurogastroenterol Motil 2012;24:1008, e542.

3. Connor FL, Di Lorenzo C. Chronic intestinal pseudo-obstruction: assessment and management. Gastroenterology 2006;130(2 suppl 1):S29S36.

4. Gabbard SL, Lacy BE. Chronic intestinal pseudo-obstruction. Nutr Clin Pract 2013;28:307-316.

5. Lauro A, De Giorgio R, Pinna AD. Advancement in the clinical management of intestinal pseudo-obstruction. Expert Rev Gastroenterol Hepatol 2015;9:197-208.

6. Roland BC, Ciarleglio MM, Clarke JO, et al. Small intestinal transit time is delayed in small intestinal bacterial overgrowth. J Clin Gastroenterol 2015;49:571-576.

7. Shimura S, Ishimura N, Mikami H, et al. Small intestinal bacterial overgrowth in patients with refractory functional gastrointestinal disorders. J Neurogastroenterol Motil 2016;22:60-68.

8. Saadi M, McCallum RW. Rifaximin in irritable bowel syndrome: rationale, evidence and clinical use. Ther Adv Chronic Dis 2013;4:71-75.

9. Menees SB, Maneerattannaporn M, Kim HM, Chey WD. The efficacy and safety of rifaximin for the irritable bowel syndrome: a systematic review and meta-analysis. Am J Gastroenterol 2012;107:28-35.
10. Grace E, Shaw C, Whelan K, Andreyev HJ. Review article: small intestinal bacterial overgrowth--prevalence, clinical features, current and developing diagnostic tests, and treatment. Aliment Pharmacol Ther 2013;38:674-688.

11. van Nood E, Vrieze A, Nieuwdorp M, et al. Duodenal infusion of donor feces for recurrent Clostridium difficile. N Engl J Med 2013;368:407415 .

12. Kassam Z, Lee CH, Yuan Y, Hunt RH. Fecal microbiota transplantation for Clostridium difficile infection: systematic review and meta-analysis. Am J Gastroenterol 2013;108:500-508.

13. Kelly CR, Kahn S, Kashyap P, et al. Update on fecal microbiota transplantation 2015: indications, methodologies, mechanisms, and outlook. Gastroenterology 2015;149:223-237.

14. Ohkubo $\mathrm{H}$, Iida $\mathrm{H}$, Takahashi $\mathrm{H}$, et al. An epidemiologic survey of chronic intestinal pseudo-obstruction and evaluation of the newly proposed diagnostic criteria. Digestion 2012;86:12-19.

15. Gasbarrini A, Corazza GR, Gasbarrini G, et al. Methodology and indications of H2-breath testing in gastrointestinal diseases: the Rome Consensus Conference. Aliment Pharmacol Ther 2009;29(suppl 1):1-49.

16. Bakken JS, Borody T, Brandt LJ, et al. Treating Clostridium difficile infection with fecal microbiota transplantation. Clin Gastroenterol Hepatol 2011;9:1044-1049.

17. Blaser AR, Starkopf J, Kirsimägi Ü, Deane AM. Definition, prevalence, and outcome of feeding intolerance in intensive care: a systematic review and meta-analysis. Acta Anaesthesiol Scand 2014;58:914-922.

18. Jones K, Mangram AJ, Lebron RA, Nadalo L, Dunn E. Can a computed tomography scoring system predict the need for surgery in smallbowel obstruction? Am J Surg 2007;194:780-783; discussion 783-784.

19. Stanghellini V, Cogliandro RF, De Giorgio R, et al. Natural history of chronic idiopathic intestinal pseudo-obstruction in adults: a single center study. Clin Gastroenterol Hepatol 2005;3:449-458.

20. Stanghellini V, Cogliandro RF, De Giorgio R, et al. Natural history of intestinal failure induced by chronic idiopathic intestinal pseudo-obstruction. Transplant Proc 2010;42:15-18.

21. Panganamamula KV, Parkman HP. Chronic intestinal pseudo-obstruction. Curr Treat Options Gastroenterol 2005;8:3-11.

22. Sabbagh C, Amiot A, Maggiori L, Corcos O, Joly F, Panis Y. Nontransplantation surgical approach for chronic intestinal pseudo-obstruction: analysis of 63 adult consecutive cases. Neurogastroenterol Motil 2013;25:e680-e686

23. Knowles CH, De Giorgio R, Kapur RP, et al. The London Classification of gastrointestinal neuromuscular pathology: report on behalf of the Gastro 2009 International Working Group. Gut 2010;59:882-887.

24. Quigley EM. Small intestinal bacterial overgrowth: what it is and what it is not. Curr Opin Gastroenterol 2014;30:141-146.

25. Jacobs C, Coss Adame E, Attaluri A, Valestin J, Rao SS. Dysmotility and proton pump inhibitor use are independent risk factors for small intestinal bacterial and/or fungal overgrowth. Aliment Pharmacol Ther 2013;37:1103-1111.

26. Lee HR, Pimentel M. Bacteria and irritable bowel syndrome: the evidence for small intestinal bacterial overgrowth. Curr Gastroenterol Rep 2006;8:305-311. 
27. Pimentel M, Soffer EE, Chow EJ, Kong Y, Lin HC. Lower frequency of MMC is found in IBS subjects with abnormal lactulose breath test, suggesting bacterial overgrowth. Dig Dis Sci 2002;47:2639-2643.

28. Barbara G, Stanghellini V, Brandi G, et al. Interactions between commensal bacteria and gut sensorimotor function in health and disease. Am J Gastroenterol 2005;100:2560-2568.

29. Grasa L, Abecia L, Forcén R, et al. Antibiotic-induced depletion of murine microbiota induces mild inflammation and changes in toll-like receptor patterns and intestinal motility. Microb Ecol 2015;70:835-848.

30. Ponziani FR, Gerardi V, Gasbarrini A. Diagnosis and treatment of small intestinal bacterial overgrowth. Expert Rev Gastroenterol Hepatol 2016;10:215-227.
31. Robinson DR, Gebhart GF. Inside information: the unique features of visceral sensation. Mol Interv 2008;8:242-253.

32. Chichlowski M, Rudolph C. Visceral pain and gastrointestinal microbiome. J Neurogastroenterol Motil 2015;21:172-181.

33. Crouzet L, Gaultier E, Del'Homme C, et al. The hypersensitivity to colonic distension of IBS patients can be transferred to rats through their fecal microbiota. Neurogastroenterol Motil 2013;25:e272-e282.

34. Pinn DM, Aroniadis OC, Brandt LJ. Is fecal microbiota transplantation (FMT) an effective treatment for patients with functional gastrointestinal disorders (FGID)? Neurogastroenterol Motil 2015;27:19-29.

35. Cogliandro RF, De Giorgio R, Barbara G, et al. Chronic intestinal pseudo-obstruction. Best Pract Res Clin Gastroenterol 2007;21:657-669. 